\title{
SUR UNE NUCLEOPHAGA PARASITE D'ENDOLIMAX NANA
}

\author{
Par E. BRUMPT et G. LAVIER
}

L'un de nous a publié en 1926 l'observation d'un malade à parasitisme intestinal complexe (Entamœba dispar, Entamoba coli, Endolimax nana, Giardia intestinalis, Blastocystis, Tænia saginata) à partir duquel put être réalisée une infestation expérimentale du chat par E. coli.

En ce qui concerne, dans ce cas plus particulièrement, la présence d'Endolimax nana, un premier examen des selles, pratiqué le 21 décembre 1925, n'avait montré que des kystes, d'ailleuis abondants, de cette amibe. Le 29 décembre, après administration d'une purgation, on pouvait en observer de très nombreuses formes végétatives, mais, fait curieux, une proportion considérable de celles-ci avaient leur noyau parasité par l'organisme qui fait l'objet de cette étude.

Ce parasite n'a pas été observé à frais, mais sur des frottis fixés au liquide de Bouin et colorés à l'hématoxyline ferrique. Sa fréquence (78 pour cent $E$. nana prises au hasard) nous a permis d'étudier les stades successifs de son évolution.

Au début de celle-ci, il offre l'aspect d'une sphérule fortement sidérophile d'environ $1 \mu$ de diamètre, entourée d'un mince halo clair et située dans le noyau entre la membrane nucléaire et le volumineux karyosome (pl. XI, 2) ; peu après on a dans le noyau plusieurs sphérules semblables (pl. XI, 3) ; le karyosome est refoulé contre la membrane et s'aplatit peu à peu (pl. XI, 4, 5) ; il n'est bientôt plus représenté que par une calotte excentrique qui se confond avec la membrane (pl. XI, 6, 10). Cependant le nombre des sphérules chromatiques augmente sans cesse ; elles sont généralement de volumes sensiblement égaux ; il est à noter cependant qu'au moment d'une grande activité de division, ces masses nucléaires peuvent présenter une certaine variation de taille (pl. XI, 8). Cette division paraît être amitotique, mais il est difficile d'être affirmatif à ce sujet ; nous en avons, en tout cas, souvent observé la fin où l'on voit les deux noyaux-fils encore unis par une desmose très chromatique (pl. XI, $5,6)$; cela explique la remarque de Brug (1926) qui n'avait pas vu la division des noyaux de sa Nucleophaga intestinalis, mais avait

Annales de Parasitologie, $\mathrm{T}$. XIII, $\mathrm{N}^{\circ} 5 .-1^{\mathrm{er}}$ septembre 1935 , p. 439-444. 
noté fréquemment une association binaire des « corps coccoïdes 》.

L'accroissement progressif de taille du parasite entrâine celui du noyau amibien et par suite de l'amibe elle-même. Cette hypertrophie, qui est de règle dans le parasitisme par Nucleophaga, peut être considérable ; le diamètre du noyau d' $E$. nana, qui est normalement de $2 \mu$ à $2 \mu, 5$, peut atteindre et même dépasser $8 \mu$; le karyosome n'est plus alors représenté que par un léger épaississement localisé de la membrane nucléaire ; les sphérules chromatiques parasitaires sont devenues très nombreuses; il n'est pas possible de déterminer avec précision leur nombre, mais on peut estimer que celui-ci est souvent bien supérieur à 200. Dans ces noyaux très hypertrophiés, la membrane nucléaire de l'amibe, amincie, paraît prête à se rompre (pl. XI, 10) ce qui, en fait, doit se produire pour libérer les spores. Nous n'avons rien observé qui pût suggérer l'existence d'une membrane sporangiale ; les spores paraissent bien être à nu contre les parois du noyau parasité. Ces spores, à maturité, se présentent sous forme de sphérules de $1 \mu$ environ de diamètre ; elles ne sont donc pas plus grosses que les masses chromatiques du début dont elles dérivent; une simple modification d'aspect s'est produite : la chromophilie centrale a diminué de telle sorte que c'est la paroi qui se colore le mieux; elle le fait d'ailleurs de façon inégale, si bien qu'elle paraît plus épaisse sur un côté que sur un autre ; dans le centre, éclairci, on peut observer de très fines granulations sidérophiles, mais rìen qui ressemble à une structure nucléaire précise.

Nöller a le premier (1) signalé brièvement, en 1921, l'existence de Nucleophaga chez Endolimax nana et Iodamœba bütschlii ; ce même auteur, dans son traité de 1922, ne donne pas de renseignements précis sur la morphologie du parasite; mais il en donne d'excellentes figures indiquant dès le début la présence des sphérules chromatiques, puis leur accroissement en nombre, leur taille restant sensiblement constante. Epstein (1922) (2), qui ne semble pas avoir connaissance de la découverte de l'auteur précédent, étudie des formations parasitaires qu'il a observées dans des amibes intestinales humaines : chez Entamœba coli, il n'a rencontré que des parasites intra-cytoplasmiques, mais il décrit un parasite intranucléaire qu'il a vu dans les formes végétatives seulement et

(1) On trouvera une revue bibliographique plus détaillée sur le genre Nucleophaga dans le récent mémoire de l'un de nous (G.L. ces Annales, p. 351).

(2) Nous n’avons pu consulter le mémoire original d'Epstein, ni voir ses figures. Nous le citons d'après le résumé allemand, dont nous devons une copie à l'obligeance du $\mathrm{D}^{r} \mathrm{C}$. A. Hoare, protozoologiste du Wellcome Bureau of Scientific Research à Londres. 
non dans les kystes des amibes "semi-parasites " du type limax (dans le texte russe, p. 73, il est dit que cette amibe est probablement identique à celle décrite par Wenyon comme Entamœba nana); l'infection débute par l'entrée dans le noyau d'un corpuscule sphérique qui se multiplie rapidement ; il n'a pu exactement observer le mode de multiplication, mais en tout cas il n'y a pas formation d'un sporange comme Dangeard l'avait décrit autrefois pour un parasite d'une amibe libre ; il n'y a pas d'enveloppe autour de la masse des sphérules dont chacune est à nu et libre ; la multiplication des parasites aboutit à un gigantisme de l'amibe et surtout du noyau qui peut contenir jusqu'à 200 ou 300 sphérules. A la fin de l'évolution, celles-ci mesurent de 1,5 à $2 \mu$ de diamètre, pouvant très exceptionnellement atteindre $3 \mu$; elles présentent un petit noyau vésiculaire pauvre en chromatine et sont entourées d'une membrane nette présentant un épaississement en croissant.

L'infection nucléaire amène la mort de l'amibe; le cytoplasme de celle-ci est d'abord détruit, puis la membrane nucléaire éclate et libère les parasites dans la lumière intestinale ; dans les kystes d'E. nana, Epstein n'a vu que des parasites intracytoplasmiques ; il aurait observé deux fois le passage du parasite du cytoplasme dans le noyau, et il pense que ce transfert doit se produire surtout au moment où l'amibe sort du kyste ; chez Entamœba coli, il n'a vu que des parasites du cytoplasme ayant un aspect analogue d'ailleurs à ceux du noyau des E. nana; il ne conclut toutefois pas à leur identité et crée, pour le parasite intranucléaire, l'espèce Nucleophaga hypertrophica.

Brug (1926), ignorant les travaux de Nöller et d'Epstein, décrit, sous le nom de Nucleophage intestinalis, un parasite nucléaire qu'il a observé chez Iodamæba bütschlii; il lui décrit le cycle suivant : une spore phagocytée par l'amibe traverse le cytoplasme et franchit la membrane nucléaire; le parasite présente alors l'aspect d'un corpuscule coccoïde, situé entre le karyosome et la membrane ; il se multiplie sous cette même forme, le nombre des granulations augmentant progressivement, cependant que le noyau s'hypertrophie et que le karyosome refoulé excentriquement se réduit à une mince calotte chromatique. Brug n'a pu voir de formes en division, mais a noté, comme nous l'avons dit plus haut, qu'en certains cas, les noyaux paraissent assemblés par paires, comme s'il y avait division binaire ; il n'a pas constaté non plus l'existence d'une enveloppe sporangiale, les parasites ne sont soutenus que par la membrane nucléaire dont l'éclatement les libérera dans le cytoplasme. Dans les spores mûres, la paroi est beaucoup plus chromophile que le centre et l'on ne peut observer de noyau net. 
En somme, à quelques détails près, nos observations sont en plein accord avec celles des auteurs précédents; notons seulement que les dimensions fournies par Epstein nous paraissent un peu fortes, tant pour le parasite, d'ailleurs, que pour l'amibe et que la présence d'un noyau vésiculeux au centre de la spore nous semble des plus douteuses. Mais il y a plein accord en ce qui concerne, d'une part, l'inexistence d'une enveloppe externe correspondant à une membrane sporangiale et, d'autre part, le mode d'évolution par multiplication constante des sphérules chromophiles sans que la formation des spores soit précédée par un stade plasmodial.

Il y a donc bien, comme l'a fait remarquer récemment l'un de nous pour les nucléophages d'amibes parasites du type limax, une évolution nettement différente de celle qu'on observe pour les Nucleophaga d'amibes libres, d'Endamœba et d'Entamœba. Peutêtre y a-t-il là plus qu'un simple caractère spécifique ; la connaissance de ces organismes inférieurs est encore trop fragmentaire pour permettre d'en juger actuellement.

Le parasite que nous avons observé chez Endolimax nana nous paraît bien être le même qu'á vu Nöller et qu'Epstein a décrit et nommé ; il nous semble bien aussi que le parasite vu par Brug chez Iodamœeba bütschlii est de même espèce et qu'ainsi Nucleophaga intestinalis Brug 1926 doive tomber en synonymie avec Nucleophaga hypertrophica Epstein 1922.

Reste un point intéressant à considérer : Epstein aurait vu, dans les formes kystiques, le parasite occuper non le noyau mais le cytoplasme. Dans nos préparations établies après purgation du sujet, il n'y avait pas de kystes et nous ne pouvons donc rien dire sur la présence ou l'absence du parasite dans ces formes, mais il a été

PLANCHE XI

Endolimax nana parasitée par Nucleophaga hypertrophica Epstein 1922. 1, Endolimax nana; individu normal (donné par comparaison) ; 2, Aspect de début du parasite nucléaire ; 3 , Multiplication des sphérules ; 4-5, Refoulement du karyosome ; 6-10, Augmentation progressive du nombre des sphérules avec hypertrophie du noyau et de l'amibe; en 6 et en 8 , divisions nucléaires ; en 10 , les spores ont pris l'aspect de maturité et la membrane nucléaire parait sur le point de se rompre ; 11, Entamœba dispar, individu normal (donné par comparaison) ; 12, Entamaba dispar, individu parasité par une Sphrrita; 13, Nucleophaga aberrante dans le cytoplasme d'E. nana; noter la différence nette de taille d'avec Sphærita. 


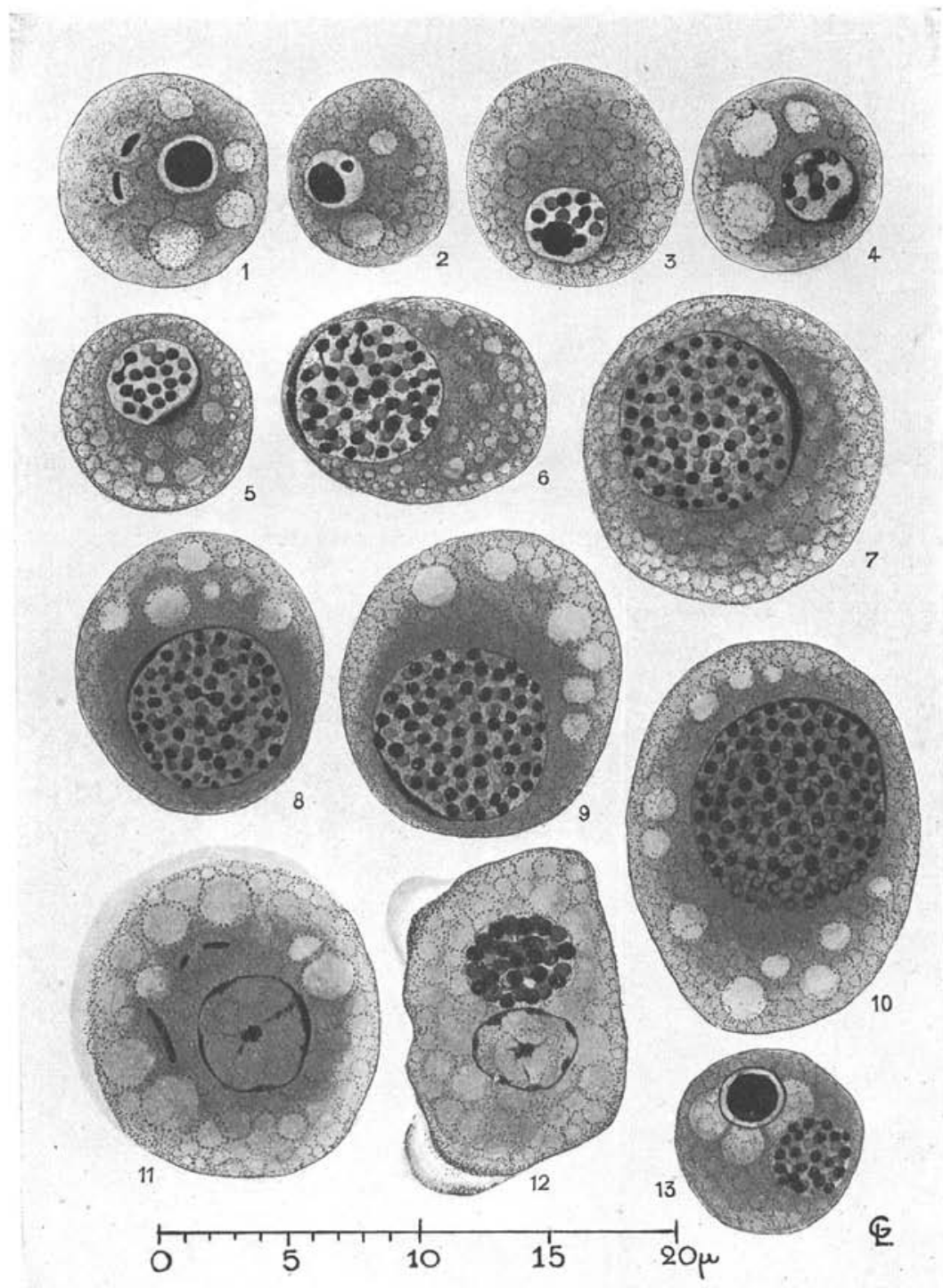


noté généralement qu'un tel parasitisme influait fortement sur l'aptitude de l'amibe à se diviser ou à s'enkyster ; si l'existence de kystes infectés n'est toutefois pas impossible, il reste à voir si ceuxci sont viables. Quant au passage supposé du parasite du cytoplasme dans le noyau au moment du dékystement, il nous paraît pure hypothèse. Le tropisme des Nucleophaga pour le noyau est en général absolu ; cependant, sur les milliers d'exemplaires parasités que nous avons observés, il nous a été donné deux seules fois, il est vrai, de rencontrer un parasite dans le cytoplasme et non dans le noyau (pl. XI, 13) ; dans les deux cas, il ne présentait qu'un petit nombre de sphérules, mais l'aspect de celles-ci se rapportait bien à celui des Nucleophaga ; il n'est pas vraisemblable que ces deux seuls exemplaires aient été parasités par des Sphærita dont les sphérules sont d'ailleurs d'un diamètre supérieur ; peut-être des infestations aussi massives que celles qui existaient dans nos préparations créent-elles, pour les nucléophages, des conditions qui leur permettent de vivre au moins transitoirement dans le cytoplasme ; peutêtre aussi s'agit-il simplement de noyaux parasités morts et phagocytés par l'amibe.

Autre fait intéressant concernant la spécificité de ces microorganismes pour leurs hôtes : moins nombretises que les Endolimax nana, mais encore très abondantes, coexistaient des Entamoba dispar (pl. XI, fig. 11) ; aucune amibe de cette espèce ne présentait de parasitisme par Nucleophaga ; par contre, beaucoup avaient, dans leur cytoplasme, des Sphærita (pl. XI, 12), alors qu'à l'exception des deux individus signalés plus haut, le cytoplasme des $E$. nana état indemne. Cette observation montre que la spécificité parasitaire de ces organismes inférieurs est beaucoup plus étroite qu'on ne le suppose généralement et que, malgré l'uniformité de leur morphologie, nous avons affaire probablement à une multiplicité d'espèces.

\section{BiBLIOGRAPHIE}

Brug (S. L.). - Nucleophage intestinalis n. sp., parasiet der Keru van Endolimax williamsi (Prow.) $=$ Endolimax bütschlii (Prow.). Meded. van den Dienst der Volksgezond. in Nederl.-Indië, IV, 1926, p. 520.

Brumpt (E.). - Infection expérimentale du chat par l'Entamœba coli Lœsch, 1875, Schaudinn emend. 1893. Ann. Parasitol., IV, 1926, p. 272.

Précis de Parasitologie, Paris, Masson, 1927, pp. 154 et 156.

EPSTEIN (H.). - [Ueber parasitische Infektion bei Darmamöben] (texte russe, résumé allemand). Archives de la Soc. russe de Protistologie, I, 1922, p. 46 . 
LAvier (G.) - Sur une Nucleophaga parasite du noyau d'Entamœba ranarum. Ann. Parasitologie hum. et comp., XIII, 1935, p. 351.

Nöller (W.). - Ueber einige wenig bekannte Darmprotozoen des Menschen und ihre nächsten Verwandten. Arch. f. Sch. und Tr. Hyg., XXV, 1921, p. 35 .

- Die wichtigsten parasitischen Prctozoen des Menschen und der Tiere. I Teil. Berlin, 1922, p. 130, fig., p. 132.

Laboraratoire de Parasilologie de la Faculté de Médecine de Paris (Directeur : Prof. E. Brumpt)

et Laboraloire de Zoologie et Parasilologie de la Faculté de Médecine de Lille (Directeur: Prof. G. Lavier) 